Research Article

\title{
Stability of Fractional Differential Equations with New Generalized Hattaf Fractional Derivative
}

\author{
Khalid Hattaf $\mathbb{D}$ \\ Centre Régional des Métiers de l'Education et de la Formation (CRMEF), 20340 Derb Ghalef, Casablanca, Morocco \\ Correspondence should be addressed to Khalid Hattaf; k.hattaf@yahoo.fr
}

Received 2 November 2021; Revised 19 November 2021; Accepted 20 November 2021; Published 2 December 2021

Academic Editor: Muhammad Shoaib Anwar

Copyright (c) 2021 Khalid Hattaf. This is an open access article distributed under the Creative Commons Attribution License, which permits unrestricted use, distribution, and reproduction in any medium, provided the original work is properly cited.

This paper aims to study the stability of fractional differential equations involving the new generalized Hattaf fractional derivative which includes the most types of fractional derivatives with nonsingular kernels. The stability analysis is obtained by means of the Lyapunov direct method. First, some fundamental results and lemmas are established in order to achieve the goal of this study. Furthermore, the results related to exponential and Mittag-Leffler stability existing in recent studies are extended and generalized. Finally, illustrative examples are presented to show the applicability of our main results in some areas of science and engineering.

\section{Introduction}

Fractional differential equations (FDEs) are recently developed in order to describe and model the dynamics of systems having memory or hereditary properties. These types of equations have been used and applied in various areas of science and engineering such as epidemiology [1], cancerology [2], viral immunology [3, 4], and viscoelastic fluid flows [5], as well as adaptive control engineering [6].

It is well known that there are two main methods to analyze the stability of ordinary differential equations (ODEs). The first one is called the Lyapunov indirect method that aims to study the local stability by means of the linearization of a system around its steady state (equilibrium point). The second method called the Lyapunov direct method consists to find or construct an appropriate auxiliary function, named a Lyapunov candidate function. Furthermore, the Lyapunov direct method provides a substantial tool for stability analysis of nonlinear systems. It can be used to determine the global dynamical behaviors of these systems without the need to solve explicitly the solutions of ODEs.

The stability of FDEs has attracted the attention of several researchers. In 2010, Li et al. [7] studied the stability of nonlinear systems of FDEs involving the Caputo fractional derivative with singular kernel [8]. They extended the
Lyapunov direct method to the case of FDEs. In the same year, Sadati et al. [9] extended the Mittag-Leffler stability theorem for fractional nonlinear systems of FDEs with delay. The stability of a class of nonlinear systems of FDEs involving the Hadamard fractional derivative [10] was investigated in [11] by using a fractional comparison principle.

The theory of the stability of FDEs involving fractional derivatives with nonsingular kernels is new, and it requires an important development in order to study the dynamical behaviors of several systems available in the literature and using such derivatives. For these reasons, the main purpose of this paper is to extend the Lyapunov direct method for systems of FDEs involving the new generalized Hattaf fractional (GHF) derivative [12], which covers the most famous fractional derivatives with nonsingular kernels existing in the literature such as the Caputo-Fabrizio fractional derivative [13], the Atangana-Baleanu fractional derivative [14], and the weighted Atangana-Baleanu fractional derivative [15].

The main advantage of using the GHF derivative is that it is a nonlocal operator and it has a nonsingular kernel formulated by the Mittag-Leffler function with a parameter different to the order of the fractional derivative. Furthermore, this operator is a weighted fractional derivative which can be used to solve various types of integral equations with elegant ways as in [16-18]. On the other hand, the novelties 
of this article are the study of the stability of FDEs with the new GHF operator by means of the Lyapunov direct method and the extension and generalization of the results related to exponential and Mittag-Leffler stability presented in [7, 19], as well as the establishment of some interesting properties and inequalities of GHF derivative in order to easily prove the Lyapunov stability theorems and construct Lyapunov candidate functions of quadratic-type, which are frequently used for demonstrating the global stability of many fractional order systems.

The outline of this paper is organized as follows. After an introductory part, Section 2 introduces the basic definitions and provides some lemmas and fundamental properties of the GHF derivative with nonsingular kernel in Caputo sense necessary to achieve the objective of this study. Section 3 is devoted to stability analysis. Finally, Section 4 presents some applications of our main results in the field of epidemiology as well as in the fractional linear systems theory.

\section{Fundamental Results}

In this section of the paper, we present the definitions and provide some fundamental results related to the GHF derivative with nonsingular kernel.

Definition 1 (see [12]). Let $\alpha \in[0,1), \quad \beta, \gamma>0$, and $f \in H^{1}(a, b)$. The GHF derivative of order $\alpha$ in Caputo sense of the function $f(t)$ with respect to the weight function $w(t)$ is defined as follows:

$$
{ }^{C} D_{a, t, w}^{\alpha, \beta, \gamma} f(t)=\frac{N(\alpha)}{1-\alpha} \frac{1}{w(t)} \int_{a}^{t} E_{\beta}\left[-\mu_{\alpha}(t-\tau)^{\gamma}\right] \frac{\mathrm{d}}{\mathrm{d} \tau}(w f)(\tau) \mathrm{d} \tau,
$$

where $w \in C^{1}(a, b), w, w^{\prime}>0$ on $[a, b], N(\alpha)$ is a normalization function obeying $N(0)=N(1)=1, \mu_{\alpha}=\alpha /(1-\alpha)$, and $E_{\beta}(t)=\sum_{k=0}^{+\infty} t^{k} / \Gamma(\beta k+1)$ is the Mittag-Leffler function of parameter $\beta$.

The GHF derivative introduced in the above definition generalizes and extends many special cases available in the literature. For instance, when $w(t)=1$ and $\beta=\gamma=1$, (1) reduced to the Caputo-Fabrizio fractional derivative [13] given by

$$
{ }^{C} D_{a, t, 1}^{\alpha, 1,1} f(t)=\frac{N(\alpha)}{1-\alpha} \int_{a}^{t} \exp \left[-\mu_{\alpha}(t-\tau)\right] f^{\prime}(\tau) \mathrm{d} \tau .
$$

When $w(t)=1$ and $\beta=\gamma=\alpha$, (1) reduced to the Atangana-Baleanu fractional derivative [14] given by

$$
{ }^{C} D_{a, t, 1}^{\alpha, \alpha, \alpha} f(t)=\frac{N(\alpha)}{1-\alpha} \int_{a}^{t} E_{\alpha}\left[-\mu_{\alpha}(t-\tau)^{\alpha}\right] f^{\prime}(\tau) \mathrm{d} \tau .
$$

Furthermore, the weighted Atangana-Baleanu fractional derivative [15], given by

$$
{ }^{C} D_{a, t, w}^{\alpha, \alpha, \alpha} f(t)=\frac{N(\alpha)}{1-\alpha} \frac{1}{w(t)} \int_{a}^{t} E_{\alpha}\left[-\mu_{\alpha}(t-\tau)^{\alpha}\right] \frac{\mathrm{d}}{\mathrm{d} \tau}(w f)(\tau) \mathrm{d} \tau
$$

is a special case of GHF derivative; it suffices to take $\beta=\gamma=\alpha$.

Considering the importance of weighted fractional derivatives to write and solve many integral equations in an elegant way, the function $w$ has been introduced in equation (1). For instance, we consider the following integral equation:

$$
\lambda \int_{0}^{t} e^{-\lambda(t-\tau)} E_{\beta}\left[-\rho(t-\tau)^{\gamma}\right] f(\tau) \mathrm{d} \tau+\int_{0}^{t} e^{-\lambda(t-\tau)} E_{\beta}\left[-\rho(t-\tau)^{\gamma}\right] f^{\prime}(\tau) \mathrm{d} \tau=\Lambda-\delta f(t)
$$

where $\lambda, \rho, \Lambda, \delta>0$. In terms of the GHF operator, this equation can be written as follows:

$$
{ }^{C} D_{a, t, w}^{\alpha, \beta, \gamma} f(t)=A-\mu f(t),
$$

where $w(t)=e^{\lambda t}, N(\alpha)=1, \alpha=\rho /(1+\rho), A=\Lambda /(1-\alpha)$, and $\mu=\delta /(1-\alpha)$. Similar to the example of HIV infection presented in [12], the solution of the above integral equation when $\gamma=\beta$ is given by

$$
\begin{aligned}
f(t)= & \frac{A}{\mu}+\frac{N(\alpha) w(0)}{a_{\alpha} w(t)}\left(f(0)-\frac{A}{\mu}\right) E_{\beta}\left(-\frac{\alpha \mu}{a_{\alpha}} t^{\beta}\right) \\
& -\frac{A N(\alpha)}{\mu a_{\alpha} w(t)} E_{\beta}\left(-\frac{\alpha \mu}{a_{\alpha}} t^{\beta}\right) * w^{\prime}(t),
\end{aligned}
$$

where $a_{\alpha}=N(\alpha)+\mu(1-\alpha)$.

In various areas of science and engineering, the method of constructing Lyapunov functions is often based on quadratic-type functions. So, we provide the following lemma that estimates the GHF derivative of these types of Lyapunov candidate functions.

Lemma 1. Let $x(.) \in I R^{n}$ be a continuously differentiable function and $P \in I R^{n \times n}$ be a symmetric positive definite matrix. Then, for any time $t \geq t_{0}$, we have

$$
{ }^{C} D_{t_{0}, t, 1}^{\alpha, \beta, \gamma}\left(x(t)^{T} P x(t)\right) \leq 2 x(t)^{T} P^{C} D_{t_{0}, t, 1}^{\alpha, \beta, \gamma} x(t) .
$$

Proof. Similar to $[19,20]$, we consider the following function:

$$
g(t)={ }^{C} D_{t_{0}, t, 1}^{\alpha, \beta, \gamma}\left(x(t)^{T} P x(t)\right)-2 x(t)^{T} P^{C} D_{t_{0}, t, 1}^{\alpha, \beta, \gamma} x(t) .
$$

Then, 


$$
\begin{aligned}
g(t) & =\frac{N(\alpha)}{1-\alpha} \int_{t_{0}}^{t} E_{\beta}\left[-\mu_{\alpha}(t-\tau)^{\gamma}\right]\left(2 x(\tau)^{T} P \dot{x}(\tau)-2 x(t)^{T} P \dot{x}(\tau)\right) \mathrm{d} \tau \\
& =\frac{N(\alpha)}{1-\alpha} \int_{t_{0}}^{t} E_{\beta}\left[-\mu_{\alpha}(t-\tau)^{\gamma}\right]\left(2 y(\tau)^{T} P \dot{y}(\tau)\right) \mathrm{d} \tau \\
& =\frac{N(\alpha)}{1-\alpha} \int_{t_{0}}^{t} E_{\beta}\left[-\mu_{\alpha}(t-\tau)^{\gamma}\right]\left(y(\tau)^{T} P y(\tau)\right)^{\prime} \mathrm{d} \tau
\end{aligned}
$$

where $y(\tau)=x(\tau)-x(t)$. Integrating by parts, we obtain

$$
\begin{aligned}
g(t)= & \left.\frac{N(\alpha)}{1-\alpha} E_{\beta}\left[-\mu_{\alpha}(t-\tau)^{\gamma}\right] y(\tau)^{T} P y(\tau)\right|_{\tau=t_{0}} ^{\tau=t} \\
& -\frac{\alpha \gamma N(\alpha)}{(1-\alpha)^{2}} \int_{t_{0}}^{t}(t-\tau)^{\gamma-1} E_{\beta, \beta+1}^{2}\left[-\mu_{\alpha}(t-\tau)^{\gamma}\right] y(\tau)^{T} P y(\tau) \mathrm{d} \tau .
\end{aligned}
$$

Since $\lim _{\tau \longrightarrow t} E_{\beta}\left[-\mu_{\alpha}(t-\tau)^{\gamma}\right] y(\tau)^{T} P y(\tau)=y(t)^{T} P y(t)$ $=0$, we have

$$
\begin{aligned}
g(t)= & -\frac{N(\alpha)}{1-\alpha} E_{\beta}\left[-\mu_{\alpha}\left(t-t_{0}\right)^{\gamma}\right] y\left(t_{0}\right)^{T} P y\left(t_{0}\right) \\
& -\frac{\alpha \gamma N(\alpha)}{(1-\alpha)^{2}} \int_{t_{0}}^{t}(t-\tau)^{\gamma-1} E_{\beta, \beta+1}^{2}\left[-\mu_{\alpha}(t-\tau)^{\gamma}\right] y(\tau)^{T} P y(\tau) \mathrm{d} \tau
\end{aligned}
$$

This follows that $g(t) \leq 0$, for all $t \geq t_{0}$, and the proof is completed.

Remark 1. It is important to note that the above lemma extends the recent results presented in Lemma 2 of [19] and Corollary 1 of [20]. Moreover, the results presented in Lemma 3.1 of [21] to estimate the Atangana-Baleanu Caputo derivative of quadratic Lyapunov functions is extended to the case of GHF derivative.

For simplicity, denote ${ }^{C} D_{a, t, w}^{\alpha, \beta, \beta}$ by $\mathscr{D}_{a, w}^{\alpha, \beta}$. By [12], the generalized fractional integral associated to $\mathscr{D}_{a, w}^{\alpha, \beta}$ is given by the following definition.

Definition 2 (see [12]). The generalized fractional integral operator associated to $\mathscr{D}_{a, w}^{\alpha, \beta}$ is defined by

$$
\mathscr{J}_{a, w}^{\alpha, \beta} f(t)=\frac{1-\alpha}{N(\alpha)} f(t)+\frac{\alpha}{N(\alpha)}^{R L} \mathscr{J}_{a, w}^{\beta} f(t),
$$

where ${ }^{R L} \mathscr{I}_{a, w}^{\beta}$ is the standard weighted Riemann-Liouville fractional integral of order $\beta$ defined

$$
{ }^{R L} \mathscr{J}_{a, w}^{\beta} f(t)=\frac{1}{\Gamma(\beta)} \frac{1}{w(t)} \int_{a}^{t}(t-\tau)^{\beta-1} w(\tau) f(\tau) \mathrm{d} x .
$$

Remark 2. The Atangana-Baleanu fractional integral operator is a particular case of (7), and it suffices to take $w(t)=$ 1 and $\beta=\alpha$.

Now, we recall an important theorem that we will need in the following. This theorem extends the Newton-Leibniz formula introduced in $[22,23]$.

Theorem 1 (see [20]). Let $\alpha \in[0,1), \quad \beta>0$, and $f \in H^{1}(a, b)$. Then, we have the following properties:

$$
\begin{aligned}
& \mathscr{J}_{a, w}^{\alpha, \beta}\left(\mathscr{D}_{a, w}^{\alpha, \beta} f\right)(t)=f(t)-\frac{w(a) f(a)}{w(t)}, \\
& \mathscr{D}_{a, w}^{\alpha, \beta}\left(\mathscr{J}_{a, w}^{\alpha, \beta} f\right)(t)=f(t)-\frac{w(a) f(a)}{w(t)} .
\end{aligned}
$$

On the contrary, we need the following results.

Lemma 2. Let $y(.) \in I R^{n}$. The solution of the following Cauchy problem

$$
\mathscr{D}_{0, w}^{\alpha, \beta} y(t)=\lambda y(t)+u(t)
$$

with initial boundary condition $y(0)=y_{0}$, is given by

$$
\begin{aligned}
y(t)= & \frac{N(\alpha) w(0) y_{0}}{a_{\alpha} w(t)} E_{\beta}\left(\frac{\alpha \lambda}{a_{\alpha}} t^{\beta}\right)+\frac{1-\alpha}{a_{\alpha}} u(t) \\
& +\frac{N(\alpha)}{\lambda a_{\alpha} w(t)} \frac{\mathrm{d}}{\mathrm{d} t} E_{\beta}\left(\frac{\alpha \lambda}{a_{\alpha}} t^{\beta}\right) *(w u)(t),
\end{aligned}
$$

where $a_{\alpha}=N(\alpha)-\lambda(1-\alpha) \neq 0$ and $\lambda \neq 0$.

Proof. By (11), we have

$$
w(t) \mathscr{D}_{0, w}^{\alpha, \beta} y(t)=\lambda w(t) y(t)+w(t) u(t) .
$$

By applying Laplace transform and using Theorem 2 in [12], we obtain

$$
\begin{aligned}
\mathscr{L}\{w(t) y(t)\}= & \frac{(1-\alpha)\left(s^{\beta}+\mu_{\alpha}\right)}{[N(\alpha)-\lambda(1-\alpha)] s^{\beta}-\lambda \alpha} \mathscr{L}\{w(t) u(t)\} \\
& +\frac{N(\alpha) w(0) y(0) s^{\beta-1}}{[N(\alpha)-\lambda(1-\alpha)] s^{\beta}-\lambda \alpha} .
\end{aligned}
$$

Then, 


$$
\begin{aligned}
\mathscr{L}\{w(t) y(t)\}= & \frac{N(\alpha) w(0) y(0)}{a_{\alpha}} \frac{s^{\beta-1}}{s^{\beta}-\left(\alpha \lambda / a_{\alpha}\right)}+\frac{1-\alpha}{a_{\alpha}} \frac{s^{\beta-1}}{s^{\beta}-\left(\alpha \lambda / a_{\alpha}\right)} s \mathscr{L}\{w(t) u(t)\} \\
& +\frac{\alpha}{a_{\alpha}} \frac{1}{s^{\beta}-\left(\alpha \lambda / a_{\alpha}\right)} \mathscr{L}\{w(t) u(t)\} \\
= & \frac{N(\alpha) w(0) y(0)}{a_{\alpha}} \mathscr{L}\left\{E_{\beta}\left(\frac{\alpha \lambda}{a_{\alpha}} t^{\beta}\right)\right\}+\frac{1-\alpha}{a_{\alpha}} \mathscr{L}\left\{E_{\beta}\left(\frac{\alpha \lambda}{a_{\alpha}} t^{\beta}\right)\right\}\left[\mathscr{L}\left\{(w u)^{\prime}(t)\right\}+w(0) u(0)\right] \\
& +\frac{1}{\lambda} \mathscr{L}\left\{\frac{\mathrm{d}}{\mathrm{d} t} E_{\beta}\left(\frac{\alpha \lambda}{a_{\alpha}} t^{\beta}\right)\right\} \mathscr{L}\{w(t) u(t)\} .
\end{aligned}
$$

The passage to the inverse Laplace gives

$$
\begin{aligned}
w(t) y(t)= & \frac{N(\alpha) w(0) y(0)}{a_{\alpha}} E_{\beta}\left(\frac{\alpha \lambda}{a_{\alpha}} t^{\beta}\right)+\frac{1-\alpha}{a_{\alpha}} w(t) u(t) \\
& +\frac{N(\alpha)}{\lambda a_{\alpha}} \frac{\mathrm{d}}{\mathrm{d} t} E_{\beta}\left(\frac{\alpha \lambda}{a_{\alpha}} t^{\beta}\right) *(w u)(t) .
\end{aligned}
$$

From integration by parts, we have

$$
\begin{aligned}
\frac{\mathrm{d}}{\mathrm{d} t} E_{\beta}\left(\frac{\alpha \lambda}{a_{\alpha}} t^{\beta}\right) *(w u)(t)= & E_{\beta}\left(\frac{\alpha \lambda}{a_{\alpha}} t^{\beta}\right)(w u)(0)-(w u)(t) \\
& +E_{\beta}\left(\frac{\alpha \lambda}{a_{\alpha}} t^{\beta}\right) *(w u)^{\prime}(t) .
\end{aligned}
$$

Hence,

$$
\begin{aligned}
y(t)= & \frac{N(\alpha) w(0) y_{0}}{a_{\alpha} w(t)} E_{\beta}\left(\frac{\alpha \lambda}{a_{\alpha}} t^{\beta}\right)+\frac{1-\alpha}{a_{\alpha}} u(t) \\
& +\frac{N(\alpha)}{\lambda a_{\alpha} w(t)} \frac{\mathrm{d}}{\mathrm{d} t} E_{\beta}\left(\frac{\alpha \lambda}{a_{\alpha}} t^{\beta}\right) *(w u)(t) .
\end{aligned}
$$

This completes the proof.
Proof. From (14), we deduce that there exists a nonnegative function $u(t)$ such that

$$
\mathscr{D}_{0, w}^{\alpha, \beta} f(t)=-\lambda f(t)-u(t) .
$$

Remark 3. By using (12), the solution of (11) can be rewritten as follows:

$$
\begin{aligned}
y(t)= & \frac{\left(\lambda y_{0}+u(0)\right) N(\alpha) w(0)}{\lambda a_{\alpha} w(t)} E_{\beta}\left(\frac{\alpha \lambda}{a_{\alpha}} t^{\beta}\right)-\frac{1}{\lambda} u(t) \\
& +\frac{N(\alpha)}{\lambda a_{\alpha} w(t)} E_{\beta}\left(\frac{\alpha \lambda}{a_{\alpha}} t^{\beta}\right) *(w u)^{\prime}(t) .
\end{aligned}
$$

Corollary 1. Let $\lambda>0$ and $f(t)$ be a function satisfying the following inequality:

$$
\mathscr{D}_{0, w}^{\alpha, \beta} f(t) \leq-\lambda f(t)
$$

Then,

$$
f(t) \leq f(0) E_{\beta}\left(\frac{-\alpha \lambda t^{\beta}}{N(\alpha)+\lambda(1-\alpha)}\right) .
$$

By applying Lemma 2, we obtain

$$
\begin{aligned}
f(t)= & \frac{N(\alpha) w(0) f(0)}{[N(\alpha)+\lambda(1-\alpha)] w(t)} E_{\beta}\left(\frac{-\alpha \lambda t^{\beta}}{N(\alpha)+\lambda(1-\alpha)}\right)-\frac{(1-\alpha) u(t)}{N(\alpha)+\lambda(1-\alpha)} \\
& +\frac{N(\alpha)}{\lambda[N(\alpha)+\lambda(1-\alpha)] w(t)} \frac{\mathrm{d}}{\mathrm{d} t} E_{\beta}\left(\frac{-\alpha \lambda t^{\beta}}{[N(\alpha)+\lambda(1-\alpha)]}\right) *(w u)(t) \\
= & \frac{N(\alpha) w(0) f(0)}{[N(\alpha)+\lambda(1-\alpha)] w(t)} E_{\beta}\left(\frac{-\alpha \lambda t^{\beta}}{N(\alpha)+\lambda(1-\alpha)}\right)-\frac{(1-\alpha) u(t)}{N(\alpha)+\lambda(1-\alpha)} \\
& -\frac{\alpha \beta N(\alpha)}{\lambda[N(\alpha)+\lambda(1-\alpha)]^{2} w(t)}\left(t^{\beta-1} E_{\beta, \beta+1}^{2}\left(\frac{-\alpha \lambda t^{\beta}}{[N(\alpha)+\lambda(1-\alpha)]}\right) *(w u)(t)\right) .
\end{aligned}
$$

Then, 


$$
f(t) \leq \frac{N(\alpha) w(0) f(0)}{[N(\alpha)+\lambda(1-\alpha)] w(t)} E_{\beta}\left(\frac{-\alpha \lambda t^{\beta}}{N(\alpha)+\lambda(1-\alpha)}\right) .
$$

Since $N(\alpha) \leq N(\alpha)+\lambda(1-\alpha)$ and $w(0) \leq w(t)$, we easily have (27).

\section{Stability Analysis}

In this section, we focus on the stability analysis of the fractional differential equations with the GHF derivative.

Consider the following fractional differential nonautonomous equation:

$$
\mathscr{D}_{0, w}^{\alpha, \beta} x(t)=f(t, x(t)),
$$

where $x(t) \in I R^{n}$ is the state variable and $f:[0,+\infty) \times \Omega \longrightarrow I R^{n}$ is a continuous locally Lipschitz function and $\Omega$ is a domain of $I R^{n}$ containing the origin $x=0$.

System (31) is said to be autonomous if $f(t, x)=f(x)$. In this case, (31) becomes

$$
\mathscr{D}_{0, w}^{\alpha, \beta} x(t)=f(x(t)) .
$$

First, we give some definitions that we will need in the following.

Definition 3. The trivial equilibrium point $x=0$ of (31) is said to be stable if, for each $\epsilon>0$, there exists a $\eta>0$ such that, for any initial condition $x\left(t_{0}\right)=x_{0}$ satisfying $\left\|x_{0}\right\|<\eta$, the solution $x(t)$ of (31) satisfies $\|x(t)\|<\epsilon$, for all $t \geq t_{0}$. Furthermore, $x=0$ is said to be asymptotically stable if it is stable and $\lim _{t \rightarrow+\infty} x(t)=0$.

Definition 4. A scalar function $V(x)$ is called a Lyapunov candidate function of autonomous system (32) if it is a positive definite in a neighborhood $U$ of the origin, i.e., $V(0)=0$ and $V(x)>0$, for all $x \in U \backslash\{0\}$. In addition, a scalar function $V(t, x)$ is a Lyapunov candidate function of nonautonomous system (31) if it is positive definite, i.e., $V(t, 0)=0$, for all $t \geq t_{0}$, and if there is a time-invariant function $V_{0}(x)$ which is positive definite such that $V(t, x) \geq V_{0}(x)$, for all $t \geq t_{0}$.

Also, we introduce the definition of stability in the Mittag-Leffler sense.

Definition 5. The trivial solution of (31) is called Mittag-Leffler stable if

$$
\|x(t)\| \leq\left[m\left(x\left(t_{0}\right)\right) E_{\beta}\left(-\lambda\left(t-t_{0}\right)^{\beta}\right)\right]^{\nu},
$$

where $t_{0}$ is the initial time, $\lambda \geq 0, v>0, m(0)=0, m(x) \geq 0$, and $m(x)$ is locally Lipschitz on $x \in I R^{n}$ with the Lipschitz constant $m_{0}$.

Remark 4. Mittag-Leffler stability generalizes the exponential stability and it implies asymptotic stability.
Theorem 2. Let $x=0$ be an equilibrium point for system (31). Let $V(t, x):[0,+\infty) \times \Omega \longrightarrow I R$ be a continuously differentiable function and locally Lipschitz with respect to $x$ such that

$$
\begin{gathered}
k\|x\|^{p} \leq V(t, x), \\
\mathscr{D}_{0, w}^{\alpha, \beta} V(t, x) \leq-q V(t, x),
\end{gathered}
$$

where $t \geq 0, x \in \Omega$, and $k, p$, and $q$ are arbitrary positive constants. Then, $x=0$ is Mittag-Leffler stable. If (34) and (35) hold globally on $I R^{n}$, then $x=0$ is globally Mittag-Leffler stable.

Proof. From (35) and according to Corollary 1, we deduce that

$$
V(t, x) \leq V(0, x(0)) E_{\beta}\left(\frac{-q \alpha t^{\beta}}{N(\alpha)+q(1-\alpha)}\right) .
$$

From (34), we obtain

$$
k\|x(t)\|^{p} \leq V(0, x(0)) E_{\beta}\left(\frac{-q \alpha t^{\beta}}{N(\alpha)+q(1-\alpha)}\right),
$$

which leads to

$$
\|x(t)\| \leq\left[m_{1}(x(0)) E_{\beta}\left(-\lambda t^{\beta}\right)\right]^{1 / p},
$$

where $m_{1}(x)=V(0, x) / k$ and $\lambda=q \alpha /(N(\alpha)+q(1-\alpha))$. Clearly, $m_{1}(0)=0$ and $m_{1}(x) \geq 0$. Since $V(t, x)$ is locally Lipschitz with respect to $x$, we deduce that $m_{1}(x)$ is locally Lipschitz on $x$. Therefore, the equilibrium $x=0$ is globally Mittag-Leffler stable.

Remark 5. Theorem 2 generalizes the result of the exponential stability presented in Theorem 2 of [19]. Indeed, it suffices to take $w(t)=1, \beta=\gamma=1$, and $k=1$.

Theorem 3. Let $x=0$ be an equilibrium point for system (31). Let $V(t, x):[0,+\infty) \times \Omega \longrightarrow I R$ be a continuously differentiable function and locally Lipschitz with respect to $x$ such that

$$
\begin{aligned}
k_{1}\|x\|^{p} & \leq V(t, x) \leq k_{2}\|x\|^{p q}, \\
\mathscr{D}_{0, w}^{\alpha, \beta} V(t, x) & \leq-k_{3}\|x\|^{p q},
\end{aligned}
$$

where $t \geq 0, x \in \Omega$, and $k_{1}, k_{2}, k_{3}, p$, and $q$ are arbitrary positive constants. Then, $x=0$ is Mittag-Leffler stable. If (39) and (40) hold globally on $I R^{n}$, then $x=0$ is globally Mittag-Leffler stable.

Proof. According to (39) and (40), we have

$$
\mathscr{D}_{0, w}^{\alpha, \beta} V(t, x) \leq-\frac{k_{3}}{k_{2}} V(t, x) .
$$

By applying Corollary 1, we have

$$
V(t, x) \leq V(0, x(0)) E_{\beta}\left(-\kappa t^{\beta}\right),
$$


where $\kappa=k_{3} \alpha /\left(k_{2} N(\alpha)+k_{3}(1-\alpha)\right)$. By (39), we obtain

$$
k_{1}\|x(t)\|^{p} \leq V(0, x(0)) E_{\beta}\left(-\kappa t^{\beta}\right) \text {, }
$$

which implies that

$$
\|x(t)\| \leq\left[m_{2}(x(0)) E_{\beta}\left(-\kappa t^{\beta}\right)\right]^{1 / p},
$$

where $m_{2}(x)=V(0, x) / k_{1}$. This completes the proof.

Remark 6. Theorem 3 extends the result of the Mittag-Leffler stability presented in Theorem 5.1 of [7] for the GHF derivative with nonsingular kernel. Moreover, the result given in Theorem 4 of [19] for Caputo-Fabrizio fractional derivative is recovered when $w(t)=1, \beta=\gamma=1$, $p=2$, and $q=1$.

\section{Applications}

In this section, we apply the main results obtained in this paper to investigate the stability of the following examples of fractional systems.

Example 1. Consider the following fractional linear system:

$$
\mathscr{D}_{0, w}^{\alpha, \beta} x(t)=A x(t)
$$

where $x(t) \in I R^{n}$ is the state variable and $A \in I R^{n \times n}$.

To establish the stability of (45), we define the Lyapunov candidate function as follows:

$$
V(t, x)=x^{T} P x,
$$

where $P \in I R^{n \times n}$ is a symmetric positive definite matrix. Hence,

$$
\lambda_{\min }(P)\|x\|^{2} \leq V(t, x) \leq \lambda_{\max }(P)\|x\|^{2},
$$

where $\lambda_{\min }(P)$ and $\lambda_{\max }(P)$ are the minimum and the maximum eigenvalues of the matrix $P$, respectively. Since $P$ is positive definite, we have $\lambda_{\text {min }}(P)>0$ and $\lambda_{\max }(P)>0$.

According to Lemma 1, the GHF derivative of the Lyapunov function $V$ along the trajectories of (45) satisfies

$$
\begin{aligned}
\mathscr{D}_{0,1}^{\alpha, \beta} V(t, x) & \leq 2 x^{T} P \mathscr{D}_{0, w}^{\alpha, \beta} x \\
& =x^{T}\left(A^{T} P+P A\right) x \\
& =-x^{T} Q x,
\end{aligned}
$$

where $Q=-\left(A^{T} P+P A\right)$. It is obvious that $Q$ is a symmetric matrix. Assume that $Q$ is a positive definite matrix and let $\lambda_{\text {min }}(Q)$ be the minimum of its positive eigenvalues. Then, we have

$$
\mathscr{D}_{0,1}^{\alpha, \beta} V(t, x) \leq-\lambda_{\min }(Q)\|x\|^{2} .
$$

By applying Theorem 3, we deduce that the trivial solution of system (45) is globally Mittag-Leffler stable under the condition that $Q$ is positive definite. This condition is satisfied when $A$ is Hurwitz, i.e., all the eigenvalues of $A$ have negative real parts.
Example 2. Consider the following fractional epidemic model:

$$
\left\{\begin{array}{l}
\mathscr{D}_{0,1}^{\alpha, \beta} S(t)=\mathscr{A}-\nu S(t)-\mathscr{F}(S(t), I(t)) I(t), \\
\mathscr{D}_{0,1}^{\alpha, \beta} I(t)=\mathscr{F}(S(t), I(t)) I(t)-(\nu+d+r) I(t), \\
\mathscr{D}_{0,1}^{\alpha, \beta} R(t)=r I(t)-\nu R(t),
\end{array}\right.
$$

where $S(t), I(t)$, and $R(t)$ are the susceptible, infected, and recovered individuals at time $t$, respectively. Here, the susceptible individuals are recruited at a constant rate $\mathscr{A}$ and become infected by effective contact with infected individuals at rate $\mathscr{F}(S, I) I$. The natural death rate in all classes is denoted by $v$, while $d$ is the death rate due to the disease. Furthermore, $r$ is the recovery rate of the infected individuals.

Obviously, the first two equations of (50) do not depend on the variable $R$. Then, model (50) can be rewritten by the following system:

$$
\left\{\begin{array}{l}
\mathscr{D}_{0,1}^{\alpha, \beta} S(t)=\mathscr{A}-\nu S(t)-\mathscr{F}(S(t), I(t)) I(t), \\
\mathscr{D}_{0,1}^{\alpha, \beta} I(t)=\mathscr{F}(S(t), I(t)) I(t)-(\nu+d+r) I(t) .
\end{array}\right.
$$

As in [24], we assume that the general incidence $\mathscr{F}$ is continuously differentiable in the interior of $I R_{+}^{2}$ and satisfies the following conditions:

$$
(H) \mathscr{F}(0, S)=0 \text {, }
$$

$$
\frac{\partial \mathscr{F}}{\partial S}(S, I)>0, \frac{\partial \mathscr{F}}{\partial I}(S, I) \leq 0, \quad \text { for all } S, I \geq 0 .
$$

It is clear that $\mathscr{E}^{0}=\left(S^{0}, 0\right)$ is the disease-free equilibrium of (51), where $S^{0}=\mathscr{A} / \nu$. Then, the basic reproduction number of (51) is defined as follows:

$$
\mathscr{R}_{0}=\frac{\mathscr{F}\left(S^{0}, 0\right)}{(\nu+d+r)},
$$

which epidemiologically represents the number of secondary infections produced by a single infected individual throughout the period of infection when all individuals are uninfected. Based on the same technique in [24], we can easily prove that model (51) has another equilibrium when $\mathscr{R}_{0}>1$.

Let $\Omega=\left\{(S, I) \in I R_{+}^{2}: S \leq S^{0}\right\}$. Assume that $\mathscr{R}_{0}<1$ and consider the following Lyapunov function:

$$
L(S, I)=\omega\left(S^{0}-S\right)+I,
$$

where $\oplus=\left(1-\mathscr{R}_{0}\right) / 2 \mathscr{R}_{0}$. Obviously, $L$ is a candidate Lyapunov function. Indeed, we have $L\left(\mathscr{E}^{0}\right)=0$ and $L(S, I)>0$, for all $(S, I) \in \Omega \backslash\left\{\mathscr{E}^{0}\right\}$. Moreover, we have

$$
\begin{aligned}
\mathscr{D}_{0,1}^{\alpha, \beta} L(S, I) & =-\oplus \mathscr{D}_{0,1}^{\alpha, \beta} S+\mathscr{D}_{0,1}^{\alpha, \beta} I \\
& \leq-\Phi \nu\left(S^{0}-S\right)-\frac{\delta\left(1-\mathscr{R}_{0}\right)}{2} I,
\end{aligned}
$$

where $\delta=v+d+r$. Hence,

$$
\mathscr{D}_{0,1}^{\alpha, \beta} L(S, I) \leq-\varrho_{1} L(S, I),
$$


where $\varrho_{1}=\min \left\{v, \delta\left(1-\mathscr{R}_{0}\right) / 2\right\}$.

Let $X=(S, I) \in I R^{2}$ with the norm $\|X\|=|S|+|I|$. Then,

$$
\varrho_{2}\left\|X-\mathscr{E}^{0}\right\| \leq L(S, I),
$$

where $\varrho_{2}=\min \{\omega, 1\}$. By applying Theorem 2, we conclude that the disease-free equilibrium $\mathscr{E}^{0}$ of (51) is Mittag-Leffler stable in $\Omega$ when $\mathscr{R}_{0}<1$.

\section{Data Availability}

No data were used to support this study.

\section{Conflicts of Interest}

The author declares that he has no conflicts of interest.

\section{References}

[1] K. R. Cheneke, K. P. Rao, and G. K. Edessa, "Application of a new generalized fractional derivative and rank of control measures on cholera transmission dynamics," International Journal of Mathematics and Mathematical Sciences, vol. 2021, Article ID 2104051, 9 pages, 2021.

[2] M. El Younoussi, Z. Hajhouji, K. Hattaf, and N. Yousfi, “A new fractional model for cancer therapy with M1 oncolytic virus," Complexity, vol. 2021, Article ID 9934070, 12 pages, 2021.

[3] M. Bachraoui and K. Hattaf, "Noura yousfi, spatiotemporal dynamics of fractional hepatitis B virus infection model with humoral and cellular immunity," in Proceedings of the International Symposium on Mathematical and Computational Biology, pp. 293-313, Springer, San Diego, CA, USA, October 2020.

[4] M. Bachraoui, M. A. Ichou, K. Hattaf, and N. Yousfi, "Spatiotemporal dynamics of a fractional model for hepatitis B virus infection with cellular immunity," Mathematical Modelling of Natural Phenomena, vol. 16, pp. 1-13, 2021.

[5] A. Rasheed and M. Shoaib Anwar, "Interplay of chemical reacting species in a fractional viscoelastic fluid flow," Journal of Molecular Liquids, vol. 273, pp. 576-588, 2019.

[6] S. Ladaci and Y. Bensafia, "Indirect fractional order pole assignment based adaptive control," Engineering Science and Technology, an International Journal, vol. 19, no. 1, pp. 518-530, 2016.

[7] Y. Li, Y. Chen, and I. Podlubny, "Stability of fractional-order nonlinear dynamic systems: Lyapunov direct method and generalized Mittag-Leffler stability," Computers \& Mathematics with Applications, vol. 59, no. 5, pp. 1810-1821, 2010.

[8] I. Podlubny, "Fractional differential equations," Mathematics in Science and Engineering, vol. 198, Academic Press, San Diego, CA, USA, 1999.

[9] S. J. Sadati, D. Baleanu, A. Ranjbar, R. Ghaderi, and T. Abdeljawad, "Mittag-leffler stability theorem for fractional nonlinear systems with delay," Abstract and Applied Analysis, vol. 2010, Article ID 108651, 7 pages, 2010.

[10] A. A. Kilbas, H. M. Srivastava, and J. J. Trujillo, Theory and Applications of Fractional Differential Equations, NorthHolland Mathematics Studies, Elsevier, Amsterdam, Netherlands, 2006

[11] G. Wang, K. Pei, and Y. Chen, "Stability analysis of nonlinear Hadamard fractional differential system," Journal of the Franklin Institute, vol. 356, no. 12, pp. 6538-6546, 2019.
[12] K. Hattaf, "A new generalized definition of fractional derivative with non-singular kernel," Computation, vol. 8, pp. 1-9, 2020.

[13] A. Caputo and M. Fabrizio, "A new definition of fractional derivative without singular kernel," Progress in Fractional Differentiation and Applications, vol. 1, pp. 73-85, 2015.

[14] A. Atangana and D. Baleanu, "New fractional derivatives with nonlocal and non-singular kernel: theory and application to heat transfer model," Thermal Science, vol. 20, no. 2, pp. 763-769, 2016.

[15] M. Al-Refai, "On weighted Atangana-Baleanu fractional operators," Advances in Differential Equations, vol. 2020, pp. 1-11, 2020.

[16] O. Agrawal, "Some generalized fractional calculus operators and their applications in integral equations," Fractional Calculus and Applied Analysis, vol. 15, no. 4, pp. 700-711, 2012.

[17] M. Al-Refai and A. M. Jarrah, "Fundamental results on weighted Caputo-Fabrizio fractional derivative," Chaos, Solitons \& Fractals, vol. 126, pp. 7-11, 2019.

[18] M. A. Bayrak, A. Demir, and E. Ozbilge, "On solution of fractional partial differential equation by the weighted fractional operator," Alexandria Engineering Journal, vol. 59, no. 6, pp. 4805-4819, 2020.

[19] N. Sene, "Stability analysis of the fractional differential equations with the Caputo-Fabrizio fractional derivative," Journal of Fractional Calculus and Applications, vol. 11, no. 2, pp. 160-172, 2020.

[20] K. Hattaf, "On some properties of the new generalized fractional derivative with non-singular kernel," Mathematical Problems in Engineering, vol. 2021, Article ID 1580396, 6 pages, 2021.

[21] M. A. Taneco-Hernández and C. Vargas-De-León, "Stability and Lyapunov functions for systems with Atangana-Baleanu Caputo derivative: an HIV/AIDS epidemic model," Chaos, Solitons \& Fractals, vol. 132, Article ID 109586, 2020.

[22] J. D. Djida, A. Atangana, and I. Area, "Numerical computation of a fractional derivative with non-local and non-singular kernel," Mathematical Modelling of Natural Phenomena, vol. 12, no. 3, pp. 4-13, 2017.

[23] D. Baleanu and A. Fernandez, "On some new properties of fractional derivatives with Mittag-Leffler kernel," Communications in Nonlinear Science and Numerical Simulation, vol. 59, pp. 444-462, 2018.

[24] K. Hattaf, A. A. Lashari, Y. Louartassi, and N. Yousfi, "A delayed SIR epidemic model with a general incidence rate," Electronic Journal of Qualitative Theory of Differential Equations, vol. 3, pp. 1-9, 2013. 